\title{
Histoire culturelle des techniques à partir du Moyen Âge
}

Émilie d'Orgeix

\section{(2) OpenEdition}

\section{Journals}

Édition électronique

URL : https://journals.openedition.org/ashp/4605

DOI : $10.4000 /$ ashp.4605

ISSN : 1969-6310

Éditeur

Publications de l'École Pratique des Hautes Études

Édition imprimée

Date de publication : 1 septembre 2021

Pagination : 337-342

ISSN : 0766-0677

\section{Référence électronique}

Émilie d'Orgeix, "Histoire culturelle des techniques à partir du Moyen Âge ", Annuaire de l'École pratique des hautes études (EPHE), Section des sciences historiques et philologiques [En ligne], 152 | 2021, mis en ligne le 14 juin 2021, consulté le 17 juin 2022. URL : http://journals.openedition.org/ashp/4605 ; DOI : https://doi.org/10.4000/ashp.4605 


\title{
HISTOIRE CULTURELLE DES TECHNIQUES À PARTIR DU MOYEN ÂGE
}

\author{
Directrice d'étude : M ${ }^{\text {me }}$ Émilie D'OrGEIX
}

Programme de l'année 2019-2020: L'ingénieur militaire et la fabrique de la ville (XVI ${ }^{e}$ $X V I I T^{e}$ siècle) : expertise, compétence et espaces partagés.

Poursuivant les réflexions engagées durant la précédente conférence, l'enquête de l'année 2019-2020 a porté sur le partage des pratiques et des espaces dans la Cité à l'époque moderne. Si le rôle de l'ingénieur comme cheville ouvrière dans la mise en défense du territoire a été mis en valeur par plusieurs chercheurs (bien qu'en réalité l'étendue de ses travaux dans des centaines de villes sous tutelle militaire reste encore à approfondir), on connait peu son investissement dans l'économie et la fabrique des villes. Pourtant, ses compétences en mécanique, en pyrotechnie, en fontainerie, en architecture et ingénierie civiles, en construction et en botanique entre autres, ont été pleinement exploitées que ce soit à l'occasion de travaux effectués pour le service du roi ou auprès des corps de villes, de collaborations avec les communautés de métiers ou d'interactions avec les cercles de sociabilité et les sphères savantes. De manière même un peu paradoxale, il y a toujours eu une difficulté à « rendre visible », au-delà de la res militaria, les projets, espaces et collaborations des ingénieurs dans des villes qu'ils ont pourtant habitées, réformées et modernisées. En cause certainement le mythe tenace de l'autonomisation de la profession d'ingénieur à partir de la fin $\mathrm{du} \mathrm{XVII}^{\mathrm{e}}$ siècle et la césure artificielle entre sphères civile et militaire à partir $\mathrm{du}$ $\mathrm{XIX}^{\mathrm{e}}$ siècle dans la société européenne. Les conférences ont tenté, par circonvolutions successives, d'infléchir cette lecture historiographique.

Se fondant sur ces questions larges et volontairement ouvertes des échanges, et du partage des compétences et des espaces, les conférences de l'année ont été subdivisées en trois thématiques, intitulées " Héritage », "Formes », "Lieux et pratiques », approfondissant des dossiers documentaires (manuscrits et publiés, textuels, iconographiques...) susceptibles d'éclairer la transversalité des cultures technique et savante à l'époque moderne. Notons cependant que le passage à l'enseignement en distanciel à partir du 15 mars 2020 a limité la dernière partie du programme consacrée à l'étude in situ des manuscrits d'ingénieurs botanistes conservés à la bibliothèque du Muséum d'histoire naturelle. Les dernières séances ont donc été consacrées à l'étude de la première grande série d'atlas urbains du troisième tiers du XVIII ${ }^{\mathrm{e}}$ siècle, aujourd'hui conservée à la bibliothèque du Service historique de la Défense.

\section{Héritage : échelle de la cour, la ville dans la ville}

L'année a débuté par une première série de conférences consacrée au rôle de l'ingénieur dans la sociabilité princière des $\mathrm{XVI}^{\mathrm{e}}$ et $\mathrm{XVII}^{\mathrm{e}}$ siècles. Figure courtisane aux connaissances et aux compétences « versatiles », l'ingénieur y a été envisagé, non pas 
comme un professionnel dont le savoir était « renfermé tel un couteau dans sa gaine » tel que le définissait Bernardino Rocca au XVI ${ }^{\mathrm{e}}$ siècle mais comme une figure publique, visible et même " externalisée ", de la mécanique de cour à l'époque moderne. C'est donc en se fondant sur cette définition large de l'ingénieur « ingénieux » au service du prince, que nous avons exploré plusieurs domaines de compétences dialogiques, propices à illustrer collaborations, échanges et controverses ${ }^{1}$. Ainsi, poursuivant les travaux de Werner Oeschlin et d'Anja Buschow sur l'architecte «metteur en scène " ${ }^{2}$, les premières séances ont été consacrées à l'activité pyrotechnique civile des ingénieurs militaires dont l'exercice répondait parfaitement, selon Amédée Frézier, «à l'état et aux inclinations des jeunes princes $"{ }^{3}$. En France (et en Angleterre), différemment de l'Italie où de grandes familles d'artificiers, telles les Ruggieri, se sont illustrées, ce sont essentiellement des ingénieurs militaires, «bombardiers », artilleurs, mineurs et sapeurs qui ont soutenu l'édition de manuels pyrotechniques. Alors que l'usage des «feux de joye » était encore rarement abordé par les ingénieurs italiens $\mathrm{du} \mathrm{XVI} \mathrm{I}^{\mathrm{e}}$ siècle, plus intéressés par leur potentiel martial ${ }^{4}$, plusieurs ouvrages y sont consacrés en France depuis le début du XVII ${ }^{\mathrm{e}}$ siècle. Ne s'inscrivant plus dans la tradition des livres de recettes, Francis Malthus (v. 1590 - apr. 1650), parfois francisé en François de Malthe, ingénieur anglais passé au service de Louis XIII, commissaire des feux et artifices et capitaine général des sapes et mines, renouvelle le genre. Son ouvrage intitulé Traité des feux artificiels pour la guerre et pour la récréation ${ }^{5}$, publié en 1629 , est réédité à plusieurs reprises au cours de la première moitié du $\mathrm{XVII}^{\mathrm{e}}$ siècle avant d'être traduit en anglais. Malthus, y présente un texte volontairement dégagé des « embarras », " drogues incognües », " mixtions » et « fatras » de ses prédécesseurs (p. 232). Comme nous avons pu le voir, l'attention y est essentiellement portée à la fabrication et au gabarit des structures portantes (architectures des feux), des carcasses (formes et fûts) et des " graines métalliques » (projectiles) permettant de calculer mathématiquement les trajectoires de tirs. Si Malthus reprend certains des modèles à usage militaire publiés en 1598 par Joseph Boillot (1546-1610), « expert ingénieux » de Langres, en charge du magasin de poudres et de salpêtres ${ }^{6}$, il les modifie et les détourne pour en faire des « feux de joye ». Cette coïncidence entre « guerre et fête », se retrouve de manière omniprésente dans les manuscrits contemporains. En atteste le Livre d'opération $d u f e u$, manuscrit conservé au département

1. Simon Werrett, Fireworks. Pyrotechnic, Arts and Sciences in European History, Chicago, University of Chicago Press, 2010.

2. Werner Oeschlin, Anja Buschow, Architecture de fête, l'architecte metteur en scène, Wavre, Mardaga, 1995.

3. Amédée Frézier, Traité des feux d'artifices pour le spectacle, Paris, Charles-Antoine Jombert, 1747, p. II.

4. Par exemple : Girolamo Cattaneo, Avvertimenti et essamini intorno a quelle cose, che richiedono a un perfetto bombardiero così circa all'artiglieria, come anco a' fuochi arteficiati di Girolamo Cataneo [...] Di nuovo ristampati, \& con somma diligenza corretti, Brescia, Thomaso Bozzola, 1567.

5. Francis Malthus, Traité des feux artificiels pour la guerre et pour la récréation..., Paris, Cardin Besongne, 1640.

6. Joseph Boillot, (1546?-1605), Modelles, artifices de feu et divers instrumens de guerre, avec les moyens de s'en prévaloir pour assiéger, battre, surprendre et deffendre toutes places..., Chaumont en Bassigny, Q. Mareschal, 1598. 
des manuscrits de la Bibliothèque nationale de France auquel nous avons accordé une séance ${ }^{7}$. Dans les conférences suivantes, nous avons poursuivi avec l'étude de l'œuvre contemporaine de Jean Appier Hanzelet (1596-1647), ingénieur et maître des feux artificiels au service des ducs de Lorraine auteur, en 1630, d'un recueil intitulé La Pyrotechnie $^{8}$. Au fil de ces premières séances, nous nous sommes ainsi attaché à définir l'action de l'ingénieur « à la ville et à la guerre ». Personnage amphibie pour reprendre le terme de Natalie Zemon-Davis ${ }^{9}$, l'ingénieur s'illustre tout autant comme opérateur de transferts techniques et culturels par sa position de formateur, de créateur et d'auteur et «courroie de transmission » entre corps professionnels. Le patronage dont il bénéficie ne l'engage pas uniquement «à hanter les Grands » mais à se positionner comme un collaborateur et un interlocuteur précieux auprès de nombreux corps professionnels. Malthus entre ainsi en conversation avec des architectes pour créer les décors et théâtres éphémères abritant ses « feux de plaisir ». Appier Hanzelet collabore avec le maître chirurgien François Thybourel à l'occasion de la publication de La pyrotechnie première version de son ouvrage de 1630 sur les feux artificiels ${ }^{10}$. Ce premier volet s'est conclu par une séance consacrée à l'ouvrage récent de Margarita-Ana Vázquez Manassero sur les réseaux de fidélisation royaux et princiers des ingénieurs de la maison d'Autriche à l'époque moderne ${ }^{11}$.

\section{Formes : les nécessités de la ville forte à la « moderne»}

Nous avons désormais poursuivi ces réflexions en élargissant notre échelle : les séances ne portant plus sur l'espace de la cour mais sur celui de la ville. Il s'est ainsi agi de travailler à la fois sur la définition d'un corpus opératoire de villes fortes soumises à l'action des ingénieurs. Durant les premières séances, nous sommes ainsi revenus sur la genèse de l'emploi du mot «place » ou parfois «place forte » grâce à l'exploration de dictionnaires historiques et géographiques: celui de Claude de Seyssel (1450?-1520) ${ }^{12}$ tout d'abord puis de Antoine du Pinet ${ }^{13}$ (v. 1510-v. 1584). Tous deux illustrent comment le terme de place s'impose à partir du milieu du $\mathrm{XVI}^{\mathrm{e}}$ siècle pour définir des villes génériquement enceintes de remparts qu'ils aient été antiques, médiévaux ou modernes. Par circonvallations successives, nous nous sommes engagés dans l'étude des « listes » de places fortes, depuis la première, datée

7. Livre d'opérations du feu. Matières pour faire poudre de canon et matières à feu, v. 1600-1650, BNF, Département des manuscrits, ms. français 2015.

8. Jean Appier Hanzelet, La pyrotechnie de Hanzelet Lorrain ou sont representez les plus rares \& plus appreuvez secrets des machines \& des feux artificiels propres pour assieger, battre, surprendre \& deffendre toutes places, Pont-à-Mousson, L. \& Gaspard Bernard, 1630.

9. Natalie Zemon-Davis, Léon l'Africain. Un voyageur entre deux mondes, Paris, Payot \& Rivages, 2014 [1 ${ }^{\text {re }}$ édition française 2007].

10. Jean Appier Hanzelet et François Thybourel, Recueil de plusieurs machines militaires et feux artificiels pour la guerre, \& recreation, Pont-à-Mousson, Charles Marchant, 1620.

11. Margarita-Ana Vázquez Manassero, El “yngenio” en palacio: arte y ciencia en la corte de los Austrias (ca. 1585-1640), Madrid, Fundación Juanelo Turriano, Ediciones del Umbra, 2018.

12. Claude de Seyssel, La grand' monarchie de France, composée par mess. Claude de Seyssel lors evesque de Marseille, \& depuis archevesque de Thurin, Paris, Galiot du Pré, 1557.

13. Antoine du Pinet, Plantz, pourtraitz et descriptions de plusieurs villes et forteresses, tant de l'Europe, Asie et Afrique, que des Indes, et terres neuves, Lyon, Jan d'Ogerolles, 1564. 
en 1543 , à celle rédigées par Vauban à l'articulation des $\mathrm{XVII}^{\mathrm{e}}$ et $\mathrm{XVIII}^{\mathrm{e}}$ siècles $^{14}$. Un document en particulier a retenu notre attention, le Manuscrit sur les places, rédigé par Vauban entre 1685 et 1705, dont le long dénombrement des places du royaume, tour à tour à renforcer, désarmer ou "rayer », dresse une typologie alternative de villes sous tutelle militaire. Elles n'y sont pas uniquement définies par leurs ouvrages mais également par les forces vives, troupes, régiments, officiers, ingénieurs qui y séjournent ${ }^{15}$. Les exemples sont multiples, englobant tant la composition et/ou la recomposition des villes (villes de garnison, places démantelées, villes soumises à double gouvernance...). L'étude de la série des "places abandonnées » conservée aux archives du Service historique de la Défense à Vincennes, nous a permis d'approfondir cette réflexion concernant l'importance de considérer la présence des forces militaires en présence dans la sélection de notre corpus ${ }^{16}$. La présence de garnisons, les résidences d'ingénieurs ou même leurs fréquentes visites dans des villes « désarmées » témoignent de la continuité des échanges et des négociations entre corps municipaux et administration militaire. En d'autres termes, ainsi que l'a souligné Hélène Noizet, « la ville [moderne] est donc à la fois singularisée comme un lieu unitaire, territorialisée comme une aire et dotée d'un agir ${ }^{17}$. La " réversibilité » des cessions militaires aux corps de ville y est d'ailleurs fréquente. Ainsi, lors du démantèlement des fortifications de la ville de Dôle peu après la conquête éclair de Louis XIV, le terrain du bastion du Pont est concédé à la ville par lettre patente du roi ${ }^{18}$. Ses fondations ne sont cependant pas détruites. Le nouvel hôpital construit sur son terrain ne doit qu'en épouser la forme sans en toucher aucune substructure de manière à pouvoir être rapidement réarmé. La présence continue de militaires en ville illustre la continuité du contrôle auquel le corps de ville était soumis même après le démantèlement officiel des villes conquises. Dans le même ordre d'idées, une autre séance a été consacrée aux villes précocement démantelées d'Orange et de Nancy. Elle a permis de consolider l'hypothèse selon laquelle les ingénieurs ont continué de participer après leur démantèlement au " commerce» de la ville : son économie, ses circulations et ses échanges, C'est donc sur ce postulat de travail que nous nous sommes engagés dans la troisième thématique de l'année portant sur l'étude des interactions, perméabilités et points de suture entre sphères militaire et civile ${ }^{19}$.

14. Etat des vivres et artilleries et municions servans à icelle, trouvez en municion es villes de frontière et places fortes de Picardie, Arthois et Boullenois..., 1501-1600, BNF, Département des manuscrits français, 5195, pièce 34, « Liste des places fortes », 1543.

15. Sébastien le Prestre de Vauban, Manuscrit sur les places, 1682-1705, SHD, Vincennes, Ms. in-fol. 11.

16. SHD, Vincennes, série des «Places abandonnées », 1VH 2211 à 1 VH 2246.

17. Hélène Noizet, «La ville au Moyen Âge et à l'époque moderne », EspacesTemps.net, 2014, https:// www.espacestemps.net/articles/la-ville-au-moyen-age-et-a-lepoque-moderne/ [consulté le 7.06.2020].

18. SHD Vincennes, SVH 699, Dole, pièce 3, [Lettre patente de Louis XIV pour la cession du bastion du Pont], 1698, acte recopié en 1704 avec ajout d'un plan manuscrit situant le bastion.

19. Gaël Rideau, Pierre Serna (dir.), Ordonner et partager la ville (XVII ${ }^{-}$-XIX ${ }^{e}$ siècle), Rennes, Presses universitaires de Rennes, 2011 (Histoire). 


\section{Lieux et pratiques : la ville forte comme espace partagé}

Nous avons dorénavant procédé par «éléments » pour affiner notre enquête en nous concentrant sur l'étude des espaces partagés dans les villes fortes : portes de ville, rues des remparts, places d'armes, esplanades « sous le château » ou devant les citadelles ainsi que terrains suburbains. Une séance a ainsi été consacrée à l'espace élargi de la «porte de ville » et de ses abords qui illustre tant une typologie architecturale qu'un ensemble d'enjeux liés, d'une part, à son exposition et à sa visibilité et, d'autre part, à la gestion et au contrôle des flux et des circulations des populations qui la franchissent. Nous nous sommes positionné depuis l'extérieur de la ville pour y étudier la porte et ses ouvrages avancés. L'intention n'était pas tant ici de déceler les variations d'un modèle qui évolue, somme toute, assez peu en dépit des efforts de Jacques Tarade (1640-1722), Simon Vollant (1622-1694), Vauban et leurs successeurs, il réside plutôt en la manière dont la porte a polarisé les débats professionnels entre architectes et ingénieurs. Nous avons ainsi étudié comment, dans les années 1680, la supervision imposée de l'architecte Jules Hardouin-Mansart (1646-1708) du dessin des portes de l'ingénieur Jacques Tarade tout d'abord à Menin puis à NeufBrisach a pu cristalliser les divergences. Au-delà du choix de la structure et de l'ornementation architectes et ingénieurs ont été prompts à se corriger mutuellement, voir à se critiquer, les premiers sous couvert du respect des fonctions défensives, les seconds forts de leur position académique. Leurs échanges illuminent tout un ensemble d'enjeux d'expertises et de positionnements professionnels comme le cas du décor l'illustre. Pour les ingénieurs, l'ornementation pouvait en être parlante mais non saillante pour raison défensive (visibilité et dans une moindre mesure flanquement) et de sécurité (risque d'éclats de pierre lors des sièges). Pour les architectes, elle devait essentiellement répondre à des critères d'économie et de codification académique. Si les collaborations autour de leur mise en œuvre illustrent les tensions qu'elles suscitent, l'étude des portes est également révélatrice de modes opératoires partagés entre corps professionnels. Nous avons pu nous rendre compte que les espaces des portes euxmêmes, échappaient souvent au contrôle de l'armée pour devenir des espaces privés, ponctuellement loués aux habitants de la ville. Nous avons ainsi poursuivi les séances par l'étude de la série des dix-sept atlas urbains réalisés dans les décennies suivant le rattachement du département des Fortifications au secrétariat d'État à la Guerre le 10 mars 1743. Cette réorganisation administrative, entraînant dans son sillage la mise en place d'une politique active de remise en ordre de la gestion des bâtiments militaires, a suscité la réalisation d'atlas de gestion du patrimoine militaire. Tous figurent en plans, coupes et élévations l'ensemble des édifices acquis ou réquisitionnés par l'armée : vieilles « casernes espagnoles », couvents et hôpitaux, hôtels Renaissance ou maisons de ville pour les officiers. Ce sont ainsi des outils précieux pour évaluer le partage des espaces dans les villes. Les légendes, souvent longues, qui complètent les planches, apportent également de nombreuses informations. Outre l'état de conservation des bâtiments ou leur configuration, elles dévoilent la multiplicité d'enjeux et de stratégies d'occupation et d'appropriation que suscite l'existence de ces vastes patrimoines immobiliers intra-muros dans des villes souvent densément peuplées. Les cas d'appropriation abondent. Ainsi, dans l'atlas des bâtiments de Termonde, l'ancien 
corps de garde situé au-dessus de la porte de Waes ainsi que les pièces de l'une des tours sont occupées par un brasseur qui en avait eu la jouissance « anciennement d'un gouverneur $»^{20}$. Quant aux salles voutées du rez-de-chaussée de la Grande Garde, elles servent désormais à la corporation des bouchers ${ }^{21}$. Envisageant l'hybridation des lieux et des usages, l'appropriation des espaces et la résilience des ouvrages et des architectures, comme autant de signes à interpréter pour saisir la place et le rôle joués par l'armée dans la fabrique urbaine d'Ancien Régime, nous avons ainsi consacré nos dernières séances à déceler les traces de partage et de convivance entre populations militaire et civile dans la ville d'Ancien Régime ${ }^{22}$.

$\mathrm{Au}$ cours de l'année, nous avons également accueilli, le mardi 19 mai 2019, Valérie Nègre, professeur d'histoire des techniques à Paris I Panthéon-Sorbonne, dont l'intervention a porté sur « Le chantier comme espace de partage professionnel ».

20. Nicolas Jamez [et Jean Eghels, dessinateur], Atlas des plans et profils des batimens militaires de la ville de Termonde levés en 1755 par Nicolas Jamez ingénieur et decinés par J. Eghels, Vincennes, SHD, bibliothèque du Génie, Atlas 156, pl. 13.

21. Ibid., pl. 26.

22. Lire à ce sujet, Judith Rainhorn et Didier Terrier (dir.), Etranges voisins. Altérité et relations de proximité dans la ville depuis le XVIII siècle, Rennes, Presses universitaires de Rennes, 2010. 